\title{
La bicicleta como alternativa para el transporte y la movilidad urbana en el municipio de Puerto Colombia (Colombia)
}

\section{The bicycle as an alternative for transport and urban mobility in the municipality of Puerto Colombia (Colombia)}

DOI: $10.17981 /$ mod.arq.cuc.18.1.2017.03

Artículo. Fecha de recepción: 24/04/2017 Fecha de aceptación: 11/09/2017

\author{
Dalmiro Rafael Garcia-Estrada \\ Universidad de la Costa, CUC (Colombia)
}

dgarcia31@cuc.edu.co

Para citar este artículo:

Garcia-Estrada, D. (2017). La bicicleta como alternativa para el transporte y la movilidad urbana en el municipio de Puerto Colombia (Colombia). MODULO ARQUITECTURA-CUC, vol. 18, no. 1, pp. 51-72. DOI: 10.17981/mod.arq.cuc.18.1.2017.03

\section{Resumen}

Este artículo analiza la necesidad que tiene la población de Puerto Colombia de un medio de transporte sostenible y alternativo para su desplazamiento interno en el área urbana del municipio. Además, se estudian las condiciones actuales de la movilidad urbana en Puerto Colombia con el fin de establecer un plan de movilidad donde se articule el transporte público, privado y peatonal bajo el concepto de movilidad sostenible. Bajo estos criterios, los desplazamientos en bicicleta se presentan como una respuesta eficaz a los problemas de movilidad y transporte encontrados. Por otra parte, la implementación de un sistema alternativo no contaminante, como el ciclismo, ayuda a minimizar los riesgos de accidentes y tasa de mortalidad vial.

Palabras clave: movilidad urbana; bicicleta; carriles bici; estacionamiento para bicicletas; calidad de vida

\section{Abstract}

This paper analyzes the need of Puerto Colombia's population for a sustainable and alternative means of transport for their internal movement in the urban area of the municipality. In addition, the current conditions of urban mobility in Puerto Colombia are studied to establish a mobility plan where public, private and pedestrian transport is articulated under the concept of sustainable mobility. Under these criteria, cycling is presented as an effective response to the mobility and transport problems encountered. On the other hand, the implementation of a non-polluting alternative system, such as cycling, helps to minimize the risks of accidents and the road mortality rate.

Keywords: Urban mobility; bicycle; cycle-lanes; bikeparking; quality of life 


\section{INTRODUCCIÓN}

Una de las grandes dificultades que afrontan cada día las ciudades a nivel mundial es la movilidad urbana. Cuando se habla de movilidad, se hace referencia a las personas que se desplazan para movilizarse de un sitio a otro y no a los medios de transporte, que son los instrumentos que facilitan la realización de estos desplazamientos (Velásquez, 2015). Es así, que diariamente se producen desplazamientos desde distintos puntos de origen a diferentes destinos que necesitan un medio o modo de transporte, a través de una infraestructura de espacio público adecuado. Algunos problemas de movilidad urbana como congestión vehicular, segregación de los espacios, accidentalidad y afectación del medio ambiente, inciden de manera directa la calidad de vida de los habitantes y formas de accesibilidad en la ciudad. Esta accesibilidad debe ser tanto en oportunidades existentes que proporcionen la seguridad y disminuyan los factores generadores de accidentes y muertes; como que actúen directamente en la mejoría de las condiciones ambientales (reduciendo por tanto, la polución y los desplazamientos de energía); que favorezca el desenvolvimiento económico (reduciendo los costos y el tiempo empleado en la producción) y que promueva la articulación entre los diferentes segmentos sociales (Velásquez, 2015).
La movilidad, como componente urbano, es importante en la planificación del territorio. El tema de la movilidad urbana, asentada dentro del campo de análisis de los sistemas de transporte, tiene sus principios en los debates sobre la ciudad, sobre la base de la planificación y ordenación urbanística. Los usuarios que se desplazan son, ante todo, actores sociales, por lo tanto, la movilidad no se reduce a un hábito, sino que se aprende, se prepara y se construye (Velásquez, 2015). Es importante resaltar que algunas formas de transporte como bicicletas y/o los medios de transportes motorizados (motocicletas, automóviles, transporte público o sistemas integrados de transportes, entre otros) facilitan la movilidad y accesibilidad en el territorio.

La movilidad y el transporte son pilares relacionados en el camino de la sostenibilidad urbana. El transporte tiene un peso considerable en el marco del desarrollo sostenible por las presiones ambientales, los efectos sociales y económicos asociados y las interrelaciones con otros sectores (Jiménez, s.f.). De esta manera, la movilidad urbana integra en su análisis las condiciones socioeconómicas, demoespaciales y sociológicas de la población y, por su parte, el análisis del transporte se circunscribe al estudio de los desplazamientos diarios por persona (Montezuma, 2003). En este sentido, la movilidad urbana y el transporte son complementarios, sin embargo, el fenómeno de la motorización, como medio de transporte, ha crecido a un ritmo acelerado, incrementando los niveles 
de inseguridad vial, principalmente en los países latinoamericanos. Esta situación ha generado que las principales ciudades tomen decisiones importantes, individuales y colectivas, tratando de abarcar simultáneamente dos fenómenos concurrentes (Velásquez, 2015).

El crecimiento vertiginoso de la población, la urbanización y el parque automotor impacta los modos de vida, la economía y el ambiente. Por ello, las investigaciones en este campo son fundamentales para orientar políticas acordes a los objetivos del desarrollo sostenible. En promedio se estima que el 13,2\% del presupuesto familiar es gastado en transporte y servicios (European Commission, 2016). Por otro lado, los problemas ambientales, particularmente los concernientes al cambio climático, hacen de la movilidad urbana y el transporte temas de especial interés por su responsabilidad en las emisiones de $\mathrm{CO}_{2}$. Según datos de la Comisión Europea, el $70 \%$ de las emisiones de $\mathrm{CO}_{2}$ provienen del sector transporte y movilidad urbana (European Commission, 2016). Esta preocupación ha motivado a los Estados miembro de la Unión Europea a desarrollar una guía para el desarrollo de una movilidad urbana sostenible. Bajo este concepto la movilidad urbana deja de estar centrada en la infraestructura para enfocarse en las necesidades de la población. De igual modo, la construcción de un plan de movilidad se hace de manera participativa y no cerrada a un círculo de expertos (European Commission, 2013).
Durante la segunda mitad del siglo XX, el número de vehículos privados motorizados tuvo un fuerte incremento al pasar de 50 a 450 millones (Lizárraga, 2006). En lo que va corrido del siglo $X X I$, los países en desarrollo poseen un escenario particular en materia de transporte. La visión de estos, centrada en la infraestructura y en los vehículos motorizados, como lo explica Montezuma (2003), acentúa la segregación y los problemas de accesibilidad y movilidad de la población pobre que se desplaza en transporte colectivo y que, en estos países, representa la mayoría de la población. Por otro lado, el mismo autor señala la prioridad que tiene el transporte individual sobre el colectivo, dando como resultado una forma urbana difusa con un consumo excesivo de espacio y bajas densidades. Siguiendo con esta línea, pueden definirse tres tipos en las formas de transporte (García, 2018):

- Transporte privado o individual, en el cual los desplazamientos son realizados en un vehículo particular.

- Transporte público, colectivo o masivo, en el cual se desplazan grandes volúmenes de personas a lo largo de corredores (ciclovías, metro, tranvías, sistemas integrados, metro cables).

- Transporte semipúblico, en el cual los desplazamientos son realizados en vehículos de características intermedias como los son las minivanes. 
En el caso de las ciudades colombianas se estima que para el año 2040, Colombia contará con 10,4 millones de vehículos para una población de 61,7 millones de habitantes, frente a los 3 millones con los que contaba en el año 2010 para 45,0 millones de habitantes. Además, según el último estudio realizado por la Red de Ciudades Cómo Vamos, en 11 municipios de Colombia se identificó que el transporte privado o individual aumentó en todas las ciudades, mientras el del transporte público, colectivo o masivo bajó considerablemente; lo verdaderamente relevante de este análisis es que, si la tendencia se mantiene en 10 años, el transporte privado (individual) continuará primando sobre el transporte público (colectivo), tal como sucede actualmente en las principales ciudades del país (García, 2018). Sumado a los problemas socioeconómicos que representa el crecimiento del parque automotor, se encuentra la accidentalidad y mortalidad en las vías. Esto configura un problema de salud a nivel mundial. En términos de estadísticas, la bicicleta tiene una tasa de mortalidad baja con relación a los vehículos, peatones y motociclistas; según la Orgnización Mundial de la Salud-OMS (2015), a nivel mundial le corresponde el $4 \%$, en la región europea el $4 \%$ y en Latinoamérica el 3\%. Según cifras del Observatorio Nacional de Seguridad Vial-ONSV (2017), la bicicleta es el

Tabla 1

Victimas en hechos de tránsito total y según condición de la víctima. Colombia. Comparativo enero - septiembre (acumulado), 2016-2017.

\begin{tabular}{|c|c|c|c|c|c|c|c|c|c|c|c|c|}
\hline & \multicolumn{6}{|c|}{ Muertos } & \multicolumn{6}{|c|}{ Lesionados } \\
\hline & \multicolumn{2}{|c|}{$\begin{array}{c}2016^{\star} \\
\text { (ene-sep) }\end{array}$} & \multicolumn{2}{|c|}{$\begin{array}{c}2017^{*} \\
\text { (ene-sep) }\end{array}$} & \multicolumn{2}{|c|}{ Variaciones } & \multicolumn{2}{|c|}{$\begin{array}{c}2016 \\
\text { (ene-sep) }\end{array}$} & \multicolumn{2}{|c|}{$\begin{array}{c}2017^{*} \\
\text { (ene-sep) }\end{array}$} & \multicolumn{2}{|c|}{ Variaciones } \\
\hline $\begin{array}{l}\text { Departamento del } \\
\text { hecho/Condición } \\
\text { de la victima }\end{array}$ & Casos & $\%$ & Casos & $\%$ & Absoluta & V\% & Casos & $\%$ & Casos & $\%$ & Absoluta & V\% \\
\hline $\begin{array}{c}\text { TOTAL } \\
\text { NACIONAL }\end{array}$ & 5.010 & $100 \%$ & 4.707 & $100 \%$ & -303 & $-6.0 \%$ & 30.691 & $100 \%$ & 27.676 & $100 \%$ & -3.015 & $-9.8 \%$ \\
\hline Peatón & 1.265 & $25,2 \%$ & 1.243 & $26,4 \%$ & -22 & $-1,7 \%$ & 6.037 & $19,7 \%$ & 5.363 & $19,4 \%$ & -674 & $-11,2 \%$ \\
\hline $\begin{array}{l}\text { Usuarios de } \\
\text { motocicleta }\end{array}$ & 2.571 & $51,3 \%$ & 2.367 & $50,3 \%$ & -204 & $-7,9 \%$ & 14.717 & $57,7 \%$ & 15.766 & $57,0 \%$ & -1.949 & $-11,0 \%$ \\
\hline $\begin{array}{c}\text { Pasajero/ } \\
\text { Acompañante }\end{array}$ & 379 & $7,6 \%$ & 385 & $8,2 \%$ & 6 & $1,6 \%$ & 3.823 & $12,5 \%$ & 3.636 & $13,1 \%$ & -187 & $-4,9 \%$ \\
\hline Conductor & 277 & $5,5 \%$ & 252 & $5,4 \%$ & -25 & $-9,0 \%$ & 1.182 & $3,9 \%$ & 1.040 & $3,8 \%$ & -142 & $-12,0 \%$ \\
\hline $\begin{array}{c}\text { Usuarios de la } \\
\text { bicicleta }\end{array}$ & 262 & $5,2 \%$ & 262 & $5,6 \%$ & 0 & $0,0 \%$ & 1.906 & $6,2 \%$ & 1.857 & $6,7 \%$ & -49 & $-2,6 \%$ \\
\hline Sin información & 256 & $5,1 \%$ & 198 & $4,2 \%$ & -58 & $-22,7 \%$ & 26 & $0,1 \%$ & 12 & $0,0 \%$ & -14 & $-53,8 \%$ \\
\hline
\end{tabular}

Fuente: Observatorio Nacional de Seguridad Vial-ONSV (2017) 
medio de transporte que presenta menos víctimas por accidentes de tránsito en Colombia, con un total de 524 entre 2016 y 2017 (Tabla 1):

Las ciudades colombianas se extienden horizontalmente a lo largo de rutas vehiculares y donde el servicio público es deficiente. Solo Medellín posee un metro con dos líneas, el metrocable y un tranvía. Bogotá, la capital, no ha superado, en 50 años, la etapa de estudios para la formulación del proyecto de metro. Cali y Barranquilla, tercera y cuarta ciudad en importancia del país, no contemplan aún planes de este tipo y centran sus expectativas en el desarrollo de redes para buses articulados. En los municipios de menor tamaño, la situación es más traumática. Con los ejemplos anteriores, es preciso centrarse en estas ciudades de menor tamaño para evitar cometer errores del pasado y proyectar un nuevo paradigma de movilidad urbana en consonancia con las necesidades de la sociedad actual. El Plan Nacional de Seguridad Vial de Colombia-PNSV (2013-2021), indica que la tasa de mortalidad por accidentes de tránsito en Colombia ha presentado una tendencia al alza; entre 2005 y 2013, 61 \% del total de fallecimientos por accidentes asociados al tránsito involucran motociclistas y peatones. Según el diagnóstico presentado en el PNSV, entre 2005 y 2014 se presentaron 1.836.373 accidentes de tránsito, en los cuales 58.121 personas perdieron la vida, constituyéndose así como la segunda causa de muerte violenta en el país.
Según lo anterior, uno de los sistemas de transporte más seguro, económico y amigable con el medio ambiente es la bicicleta. La bicicleta hace parte de los medios de transporte en la movilidad sustentable. La movilidad sustentable pasó a ser un tema de gran relevancia para planificar los sistemas de movilidad urbana, ya que es un modelo que promueve la utilización de diferentes medios de transporte que son amigables con el medio ambiente, incluyentes y accesibles (López, 2018).

Como estudio de caso, se escogió el municipio de Puerto Colombia, ubicado al norte del país, dentro del área metropolitana de la ciudad de Barranquilla, y fue creado mediante ordenanza departamental No. 028 del 10 de diciembre de 1981. El municipio cuenta con 29 kilómetros de vías, en su mayoría (62\%) localizadas en el casco urbano. Según el Plan Básico de Ordenamiento Territorial-PBOT (2017), el $41 \%$ del total de vías son utilizadas por el transporte público, lo cual significa que el $59 \%$ de vías restantes es utilizada por el transporte informal (mototaxismo); asimismo, existe un déficit de $29 \mathrm{Km}$ si se toman como indicador los $40 \mathrm{Km}$ recomendados por el Banco Interamericano de Desarrollo-BID (Uninorte, 2015), para ciudades como Puerto Colombia. La motocicleta es el automotor que predomina en el municipio de Puerto Colombia, donde transitan 680 motocicletas, tanto para transporte colectivo informal como para uso particular privado. El transporte público formal es brindado por buses pertenecientes a empresas privadas. Estos automotores, sumados a los vehículos particulares, generan múltiples problemas de congestión vehicular, acciden- 
talidad, contaminación atmosférica, sonora y visual. Todo esto conlleva a plantear alternativas de movilidad urbana sostenible que impacten positivamente en la calidad de vida de los habitantes al tiempo que se mejora la economía, el atractivo turístico y las condiciones ambientales del municipio. Incentivar el tráfico peatonal y alternativo con bicicletas tiene efectos importantes en la reducción de los factores de contaminación ambiental, sonora y de emisión de partículas generadas por los sistemas de transportes motorizados (Rojas y Osorio, 2013). La implementación de sistemas de bicicletas para los habitantes y turistas de Puerto Colombia ayudará a disminuir los índices de accidentalidad y, de igual forma, se desestimulará el uso de la motocicleta que es el automotor que tiene el mayor porcentaje de accidentalidad y mortalidad, según la OMS.

El problema de movilidad urbana en el municipio de Puerto Colombia cada día se acelera por el crecimiento del parque automotor de motocicletas y la falta de control por parte de las autoridades locales y departamentales.

En los hechos asociados al tránsito, donde el motociclista ha estado involucrado, un factor determinante ha sido el crecimiento del parque automotor de motocicletas, que para el año 2011 ya era equiparable con el de los automóviles, con un total de matrículas correspondientes a 3.030.317 en motocicletas y a 3.006.091 en automóviles respectivamente (Instituto de Medicina Legal y Ciencias Forenses; Fondo de Prevención Vial, 2012).
Esta cifra demuestra el alto impacto económico y social que tiene hoy día este tipo de vehículo, ya sea por su utilización como medio de transporte principal (72.5\%), como medio de transporte alternativo (4\%) o como elemento de trabajo (21,6\%) (Comité de Ensambladoras de Motos Japonesas, 2012). Además de esto, sirve para que sus usuarios mejoren los tiempos de viaje, disminuyan sus gastos de transporte, sean más competitivo a nivel laboral; en resumen, "permite" una mejora en la calidad de vida (PNSV 2013-2021, p. 10).

De esta manera, en este artículo se analizan las condiciones actuales de la movilidad urbana en Puerto Colombia con el fin de establecer un plan de movilidad donde se articule el transporte público, privado y peatonal bajo el concepto de movilidad sostenible. Bajo estos criterios, los desplazamientos en bicicleta se presentan como una respuesta eficaz a los problemas expuestos precedentemente. En términos económicos, la bicicleta presenta ventajas significativas en costos de adquisición, mantenimiento y gastos de estacionamiento. Una bicicleta puede ser 200 veces más económica que un automóvil (Suero, 2010). Con todo esto, la bicicleta es una alternativa para el desplazamiento que puede aligerar la carga de vehículos en las vialidades. Es una práctica ecológica porque promueve la disminución de $\mathrm{CO}_{2}$, de igual manera, es accesible, de fácil manejo y un medio de transporte barato que mejora la salud física y emocional. Sin embargo, esta alter- 
nativa requiere de mayor atención por parte de las autoridades encargadas de planificar la movilidad en las grandes urbes, ya que los carriles exclusivos son insuficientes, los sistemas de acceso público son limitados y de alto costo para un sector de la población, no hay suficientes espacios para estacionar las bicicletas o guardarlas, además, existe una desigualdad de espacios para transitar y no se les da prioridad en cuestión de seguridad vial a los ciclistas (López, 2018). Por tal motivo, se analizan las estrategias para incentivar el uso de la bicicleta como un medio alternativo de transporte no contaminante y orientado a los habitantes para el municipio de Puerto Colombia. A largo plazo, se espera motivar a otras municipalidades a implementar eficazmente este medio de transporte y, así, reestablecer el derecho a la movilidad, en especial a la población de escasos recursos.

\section{Caracterización y MÉTOdos}

Área de investigación. Puerto Colombia es un municipio localizado frente al mar Caribe en la costa norte colombiana. Específicamente, se encuentra a $10^{\circ} 59^{\prime}$ de latitud norte y $74^{\circ} 50^{\prime}$ de longitud este, a una altitud de $12 \mathrm{msnm}$. Tiene una extensión aproximada de $93 \mathrm{~km}^{2}$. La topografía se caracteriza por ser plano en un $85 \%$. Su clima tropical presenta una temperatura media de $27,8^{\circ} \mathrm{C}$, cuyas extremas van de $25,7^{\circ} \mathrm{C}$ durante el mes de julio a $29,9^{\circ} \mathrm{C}$ durante el mes de febrero.
Puerto Colombia es una ciudad con una población pequeña. Administrativamente, pertenece al departamento del Atlántico, ocupando el puesto 5 en importancia. Hace parte del Área Metropolitana de la ciudad de Barranquilla. Su población asciende a 23.400 habitantes con una densidad de la población de 261,6 hab/ha. Método para el análisis de los componentes de la movilidad urbana en Puerto Colombia. El análisis de la movilidad urbana y el transporte en Puerto Colombia se enfocó en tres componentes principales: i) el diagnóstico de la red vial, ii) la identificación de los problemas de movilidad urbana y iii) el estudio del espacio público y su utilización.

La primera etapa del análisis consistió en el estudio de la documentación existente referente a la problemática a nivel internacional y nacional. Del mismo modo se analizaron las políticas en las que se circunscribe el municipio, como son el Plan Básico de Ordenamiento Territorial (PBOT, 2017), el Plan de Desarrollo de Puerto Colombia 2016-2019 (Concejo Municipal de Puerto Colombia, 2016), el Plan Nacional de Seguridad VialPNSV 2013-2021 (Concejo Colombiano de Seguridad; Ministerio de Transporte, 2013) y el Sistema de Ciudades (Banco Mundial; DPN, 2012).

La segunda etapa de la investigación consistió en el trabajo de terreno que permitió realizar el diagnóstico del estado de la red vial, el espacio público y los modos de movilización. Para ello se realizaron visitas de observación directa en campo. 
Durante el trabajo de campo se identificaron los puntos críticos de accidentalidad. Además, se ubicaron, a través de mapas, los centros de distribución y concentración de los automotores de servicio público del municipio. De igual forma, se analizaron los efectos que provocan la movilidad urbana sobre el medio ambiente (ruido, contaminación y accidentes).

El proceso culmina con la elaboración de unas propuestas para implementar el uso de la bicicleta como medio de transporte sostenible en el municipio.

\section{RESUltados y DISCUSIÓN}

Diagnóstico de la red vial. La red vial del municipio de

Puerto Colombia cuenta actualmente con 29 kilómetros de vías, de los cuales 18 kilómetros pertenecen al área urbana y 11 al área rural. De acuerdo con lo reportado por la Secretaría de Tránsito, aproximadamente 12 kilómetros de las vías del municipio están dedicados de forma exclusiva al transporte público y no se tiene información respecto a las vías preferenciales para bicicletas y peatonales (Uninorte, 2015, p. 19).

De acuerdo con el Banco Interamericano de Desarrollo, BID, un municipio como Puerto Colombia debería contar como mínimo 40 kilómetros de vía de uso exclusivo para el transporte público. Al analizar estas cifras, se observa que existen retos significativos en términos de movilidad en el municipio.

\begin{abstract}
Sumado a esto, el municipio tiene dos vías arteriales importantes que le facilitan la comunicación con las capitales Barranquilla y Cartagena para la movilidad tanto de la población como de bienes y mercancías, de acuerdo con los datos del expediente municipal de 2013. La velocidad promedio que se reporta en las vías del municipio por parte de la Secretaría de Gobierno del municipio es de 40 kilómetros por hora. De acuerdo con las referencias de la metodología ICES, la velocidad mínima para evitar la congestión vehicular en horas pico es de 30 kilómetros por hora (Uninorte, 2015, p. 19).
\end{abstract}

Identificación de los problemas de movilidad urbana. En el municipio de Puerto Colombia coexisten diferentes medios de transporte: motos, triciclos móviles ${ }^{1}$, bicicletas, automóviles y buses. El medio de transporte que predomina, tanto para uso privado como colectivo, es la motocicleta. Las motocicletas que prestan servicio de transporte se encuentran ubicadas en su mayoría en 22 puntos de distribución denominados "nevadas" (tabla 2). Estas nevadas se encuentran cerca a sitios estratégicos como la plaza central, los puntos de llegada de los buses urbanos, tiendas, puesto de salud, colegios, entre otros (Fig. 1). En general, el municipio de Puerto Colombia presenta un número de 600 motocicletas (329 en nevadas y 271 circulan por todo el municipio).

${ }^{1}$ Vehículos no motorizados de 3 ruedas accionados con el esfuerzo físico del conductor por medio de pedales. 


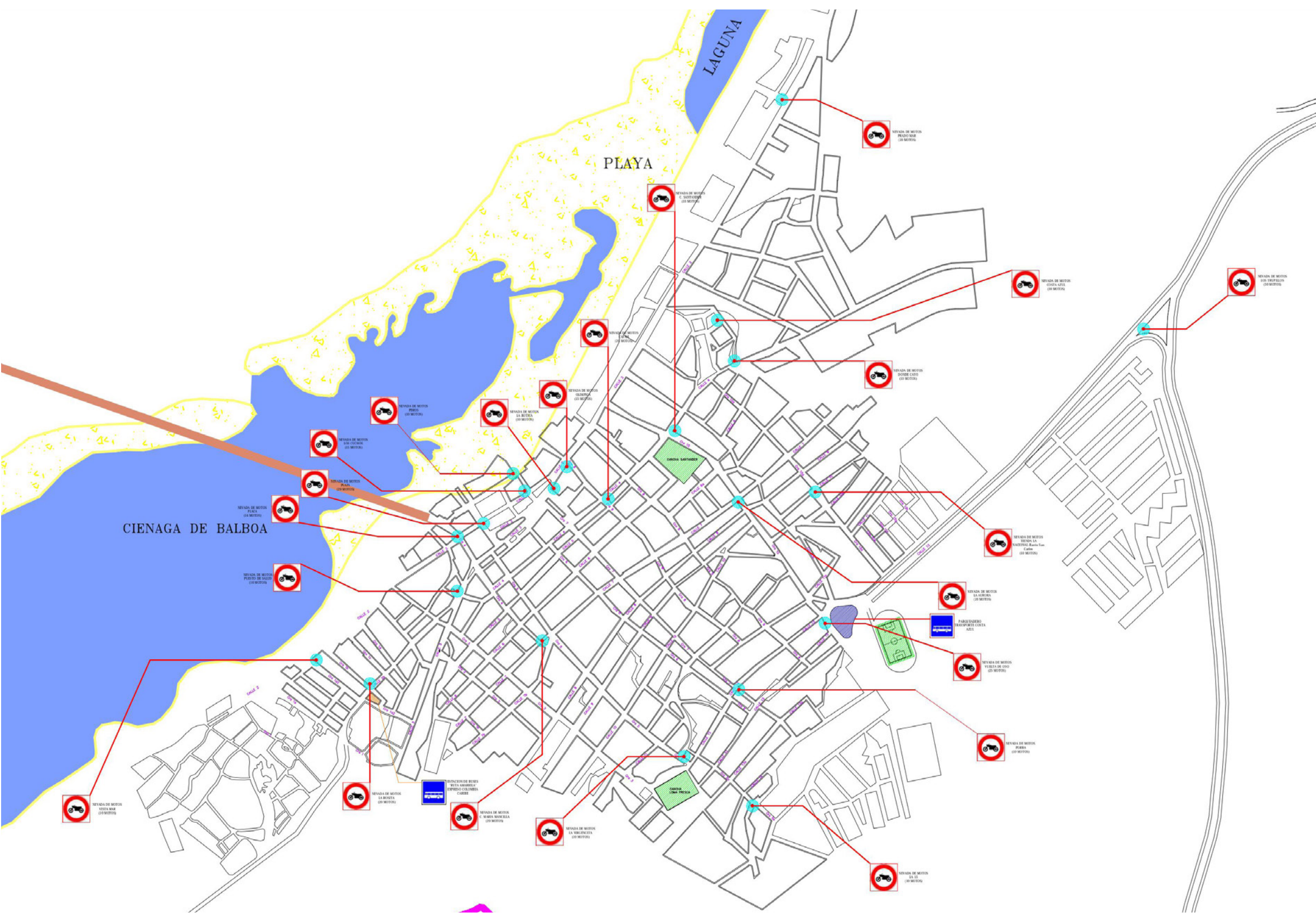

Fig. 1. Plano de nevadas de motos y buses.

Fuente: Elaboración propia con base en datos del PBOT, 2017. 
Tabla 2

Listado nevadas de motos y Buses.

\begin{tabular}{|c|c|c|c|}
\hline$N$ & $\begin{array}{l}\text { Descripción } \\
\text { de Nevada }\end{array}$ & $\begin{array}{l}\text { Cantidad } \\
\text { de motos }\end{array}$ & Buses \\
\hline 1 & La 15 & 10 & \\
\hline 2 & Porra & 10 & \\
\hline 3 & La Virgencita & 10 & \\
\hline 4 & La Nacional & 10 & \\
\hline 5 & Costa Azul & 10 & \\
\hline 6 & Donde Cayo & 10 & \\
\hline 7 & Santander & 10 & \\
\hline 8 & La Aurora & 20 & \\
\hline 9 & Pradomar & 20 & \\
\hline 10 & Los Pimos & 10 & \\
\hline 11 & Los Chuchos & 15 & \\
\hline 12 & La Bótica & 10 & \\
\hline 13 & La Olímpica & 15 & \\
\hline 14 & Cafam & 20 & \\
\hline 15 & Vuelta de Oso & 25 & \\
\hline 16 & María Mancilla & 20 & \\
\hline 17 & La Rosita & 20 & \\
\hline 18 & Puesto de Salus & 10 & \\
\hline 19 & Vista Mar & 10 & \\
\hline 20 & Plaza Principal 1 & 20 & \\
\hline 21 & Plaza Principal 2 & 14 & \\
\hline 22 & Los Trupillos & 30 & \\
\hline 23 & Buses de Costa Azul & & 45 \\
\hline \multirow[t]{2}{*}{24} & $\begin{array}{l}\text { Buses de Expreso } \\
\text { Puerto Colombia }\end{array}$ & & 35 \\
\hline & TOTAL & 329 & 80 \\
\hline
\end{tabular}

Fuente: Elaboración propia.
Sumado a todo lo anterior, se encuentra la creciente demanda de medios de transporte para los desplazamientos de la población hacia la zona urbana de la ciudad de Barranquilla. La movilidad de Puerto Colombia se condiciona y se determina por diversos flujos y aspectos de la dinámica urbana del área metropolitana de la ciudad de Barranquilla. La infraestructura de transporte, la definición de la trama vial y el uso que se les da son otro pilar fundamental de la movilidad. Sin embargo, el sentido común puede indicar que la solución del problema es simplemente construir y ampliar vías, pero la experiencia demuestra que incrementar la oferta vial sólo resuelve el problema a corto plazo, ya que se incentiva el uso del automóvil y al poco tiempo las vías estarán nuevamente congestionadas (Cantillo, 2011).

En el municipio solo prestan el servicio de transporte público dos empresas de buses: Costa Azul, que tiene 45 buses y hace un recorrido por el casco urbano de 4,2 kilómetros, y Expreso Puerto Colombia, que cuenta con una flota de 35 buses y recorre 3,8 kilómetros. En total circulan 80 buses localizados en dos nevadas y con una longitud de recorrido en las vías de 8 kilómetros (Fig. 2).

En contraste, la bicicleta no es utilizada con frecuencia por varios motivos: la infraestructura vial no está adecuada para el tránsito y existe una percepción por parte de los ciudadanos que ven 


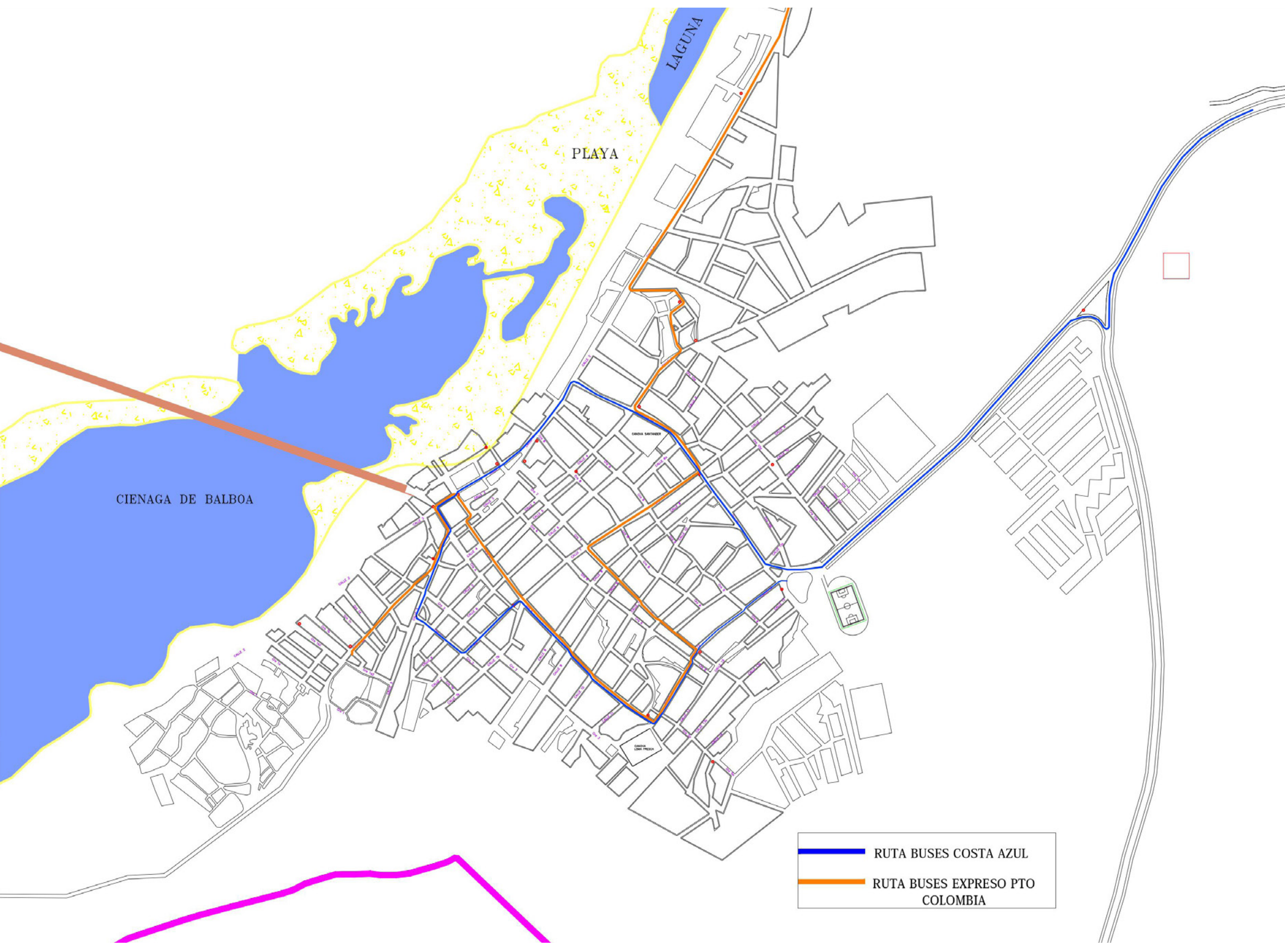

Fig. 2. Plano del recorrido de los buses urbanos.

Fuente: elaboración propia con base en datos del PBOT, 2017. 
en las motocicletas su medio de transporte más rápido para llegar a su lugar de destino. Otra alternativa particular son los bici-coches y en total existen una veintena de estos que prestan el servicio de transporte.

Al revisar el Plan Básico de Ordenamiento Territorial (PBOT, 2017) y el Plan de Desarrollo 2016-2019 (Concejo Municipal de Puerto Colombia, 2016), se aprecia que existe un interés por parte de la administración en la implementación de un sistema de ciclo-rutas que se interconecte con el espacio público. Estas alternativas son de gran apoyo para hacer de la bicicleta el medio de transporte alternativo no contaminante que se convertirá en más utilizado por los ciudadanos y turistas.

Espacio público y su utilización. Según el PBOT (2000-2012), el municipio cuenta con 14 parques y zonas verdes, 3 plazas públicas y 10 canchas deportivas (Fig. 3) para una población de 27.837 habitantes (Departamento Administrativo Nacional de Estadística-DANE, 2005). Tras la sumatoria de superficies de los diferentes espacios públicos con que cuenta el municipio, se obtiene un área de $32.300 \mathrm{~m}^{2}$. El porcentaje de espacio público por habitantes resultante es de $1,0 \mathrm{~m}^{2} / \mathrm{hab}$. No se cuenta con información sobre la adecuación extensión o estado de la infraestructura. Sin embargo, la comunidad manifestó que los escenarios se encuentran en mal estado y no satisfacen sus necesidades, por lo cual se convierte en una oportunidad de mejora como una herramienta para afianzar el desarrollo cultural y el sentido de pertenencia de la población hacia el municipio (Uninorte, 2015, p. 18).

De igual forma, el municipio no cuenta con andenes suficientes para los peatones $y$, en especial, para la población en situación de discapacidad. Se observa que los andenes en su mayoría son acogidos por los propietarios de las viviendas y los invaden con rejas o escalones, evitando la libre circulación de los ciudadanos por el espacio público (Fig. 4).

Propuesta para la implementación de una interfaz urbana. Las nevadas de buses y motocicletas, como puntos conectores de los medios de transporte del municipio, pueden convertirse en interfaces del espacio público, permitiendo el equilibrio de los sistemas de movilización. En este caso, las nevadas podrían ser los puntos de concertación de los medios de transporte: motocicletas y bicicleta, creando así, una interfaz urbana. Para que exista una interfaz, tiene que ser posible la relación de dos o más componentes (que compone o entra en composición de un todo) o entidades (colectividad considerada como unidad) (Reyes, 2018). Las nevadas, desde este contexto, se pueden replantear como estacionamientos para bicicletas (de uso público), sin dejar de lado, su antigua función de punto de concertación para las motocicletas. Esta idea la plantearemos profundamente más adelante. 


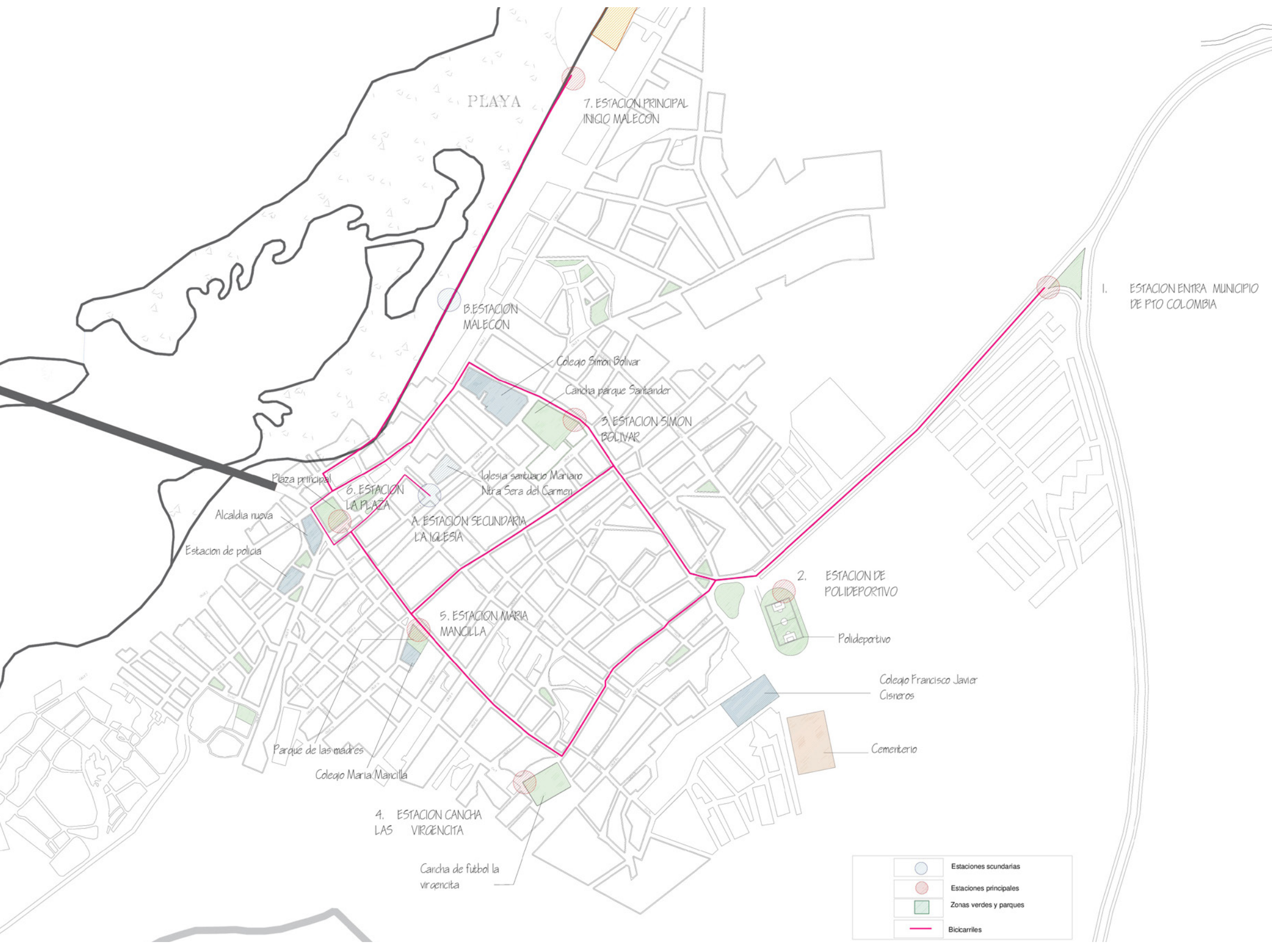

Fig. 3. Plano de espacios públicos.

Fuente: Elaboración propia con base en datos del PBOT, 2017. 


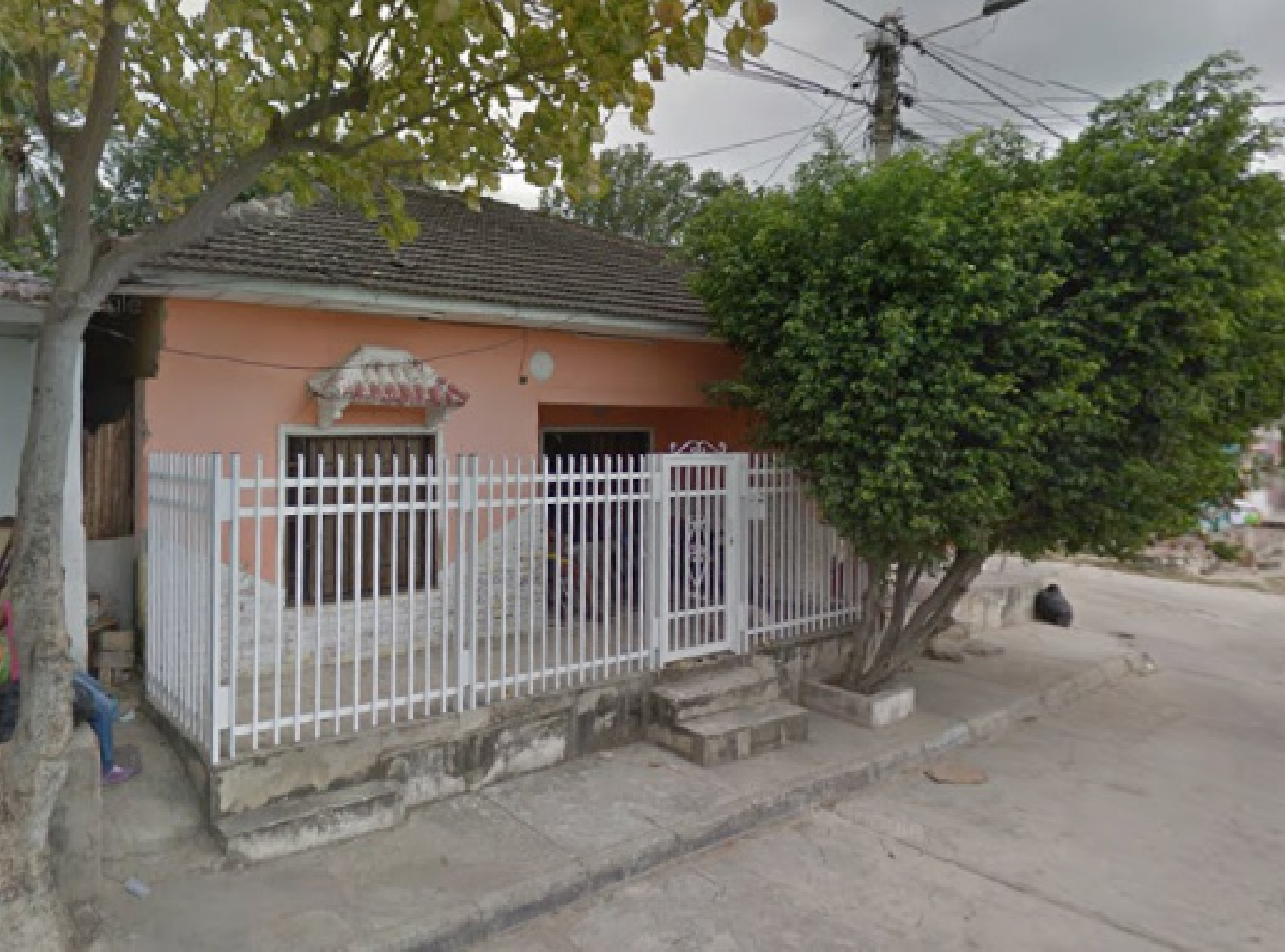

Fig. 4. Espacio público (andén) invadido en el municipio de Puerto Colombia.

Fuente: Google Street View. 
Creación de un sistema de bicicletas públicas (estaciones, ciclo-carriles, bicicletas, bici-parqueaderos). La utilización de la bicicleta como un medio de transporte alternativo sostenible en el municipio de Puerto Colombia requiere de unas intervenciones en diferentes componentes de la estructura urbana, como lo es la red vial, el espacio público y la regulación del transporte informal (mototaxismo). La propuesta es implementar estaciones principales y secundarias que se interconecten con las redes de ciclo-carriles, los cuales son carriles segregados de las vías vehiculares para facilitar el desplazamiento de los ciclistas, los cuales se separan del carril vehicular por medio de separadores tipo tachones plásticos, ayudando a delimitar los ciclo-carriles y evitar que sean invadidos por automotores. Esta interconexión es lo que se ha mencionado anteriormente como interfaz urbana del espacio público, permitiendo el equilibrio y comunicación de los distintos medios de transporte agrupado en un sistema de movilidad y red vial. La interfaz urbana, como moderadora del espacio público, es donde ocurre y se desarrolla la interacción y el intercambio, en consecuencia, su definición física, formal y funcional, en términos de articulación, debería atender y tender a la accesibilidad, a la complementariedad y la integración, permitiendo, por medio de su configuración interna y externa, los usos que definen a las entidades vinculadas en un sentido de relación y continuidad más no de unión espacial
(Reyes, 2018). Esto es lo que se buscaría con la implementación de las estaciones interconectadas de ciclo-carriles.

La malla que se diseñó obedece a las necesidades de movilidad urbana que presenta el municipio, así que se proyectaron 4,8 Km de ciclo-carriles. El ancho de los ciclo-carriles bidireccionales es de 2,0 m; se localizaron 9 estaciones de bicicletas en puntos estratégicos de espacios públicos, como plazas, parques y colegios (7 principales y 2 secundarias).

Tabla 3

Número de estaciones,

bici-parqueaderos y bicicletas.

\begin{tabular}{|c|c|c|c|c|}
\hline$N$ & Estaciones & $\begin{array}{c}\text { Bici- } \\
\text { parqueaderos }\end{array}$ & $\begin{array}{l}\text { Número } \\
\text { de } \\
\text { bicicletas }\end{array}$ & $\begin{array}{c}\text { Área } \\
\mathrm{m}^{2}\end{array}$ \\
\hline 1 & $\begin{array}{c}\text { La } \\
\text { Virgencita }\end{array}$ & 40 & 40 & 5.029 \\
\hline 2 & $\begin{array}{c}\text { María } \\
\text { Mancilla }\end{array}$ & 15 & 15 & 573 \\
\hline 3 & $\begin{array}{c}\text { Plaza } \\
\text { Principal }\end{array}$ & 30 & 30 & 2.700 \\
\hline 4 & $\begin{array}{l}\text { Principal } \\
\text { Malecón }\end{array}$ & 40 & 40 & 3.100 \\
\hline 5 & Santander & 30 & 30 & 6.900 \\
\hline 6 & $\begin{array}{c}\text { Acceso Pto. } \\
\text { Colombia } \\
\text { Vía al mar }\end{array}$ & 15 & 15 & 520 \\
\hline 7 & $\begin{array}{c}\text { Poli- } \\
\text { deportivo }\end{array}$ & 60 & 60 & 7.233 \\
\hline 8 & $\begin{array}{l}\text { Secundaria- } \\
\text { Iglesia }\end{array}$ & 15 & 15 & 580 \\
\hline 9 & $\begin{array}{c}\text { Secundaria- } \\
\text { Malecón }\end{array}$ & 10 & 10 & 350 \\
\hline & TOTAL & 255 & 255 & 26.985 \\
\hline
\end{tabular}

Fuente: Elaboración propia. 
LA BICICLETA COMO ALTERNATIVA PARA EL TRANSPORTE Y LA MOVILIDAD

URBANA EN EL MUNICIPIO DE PUERTO COLOMBIA (COLOMBIA)

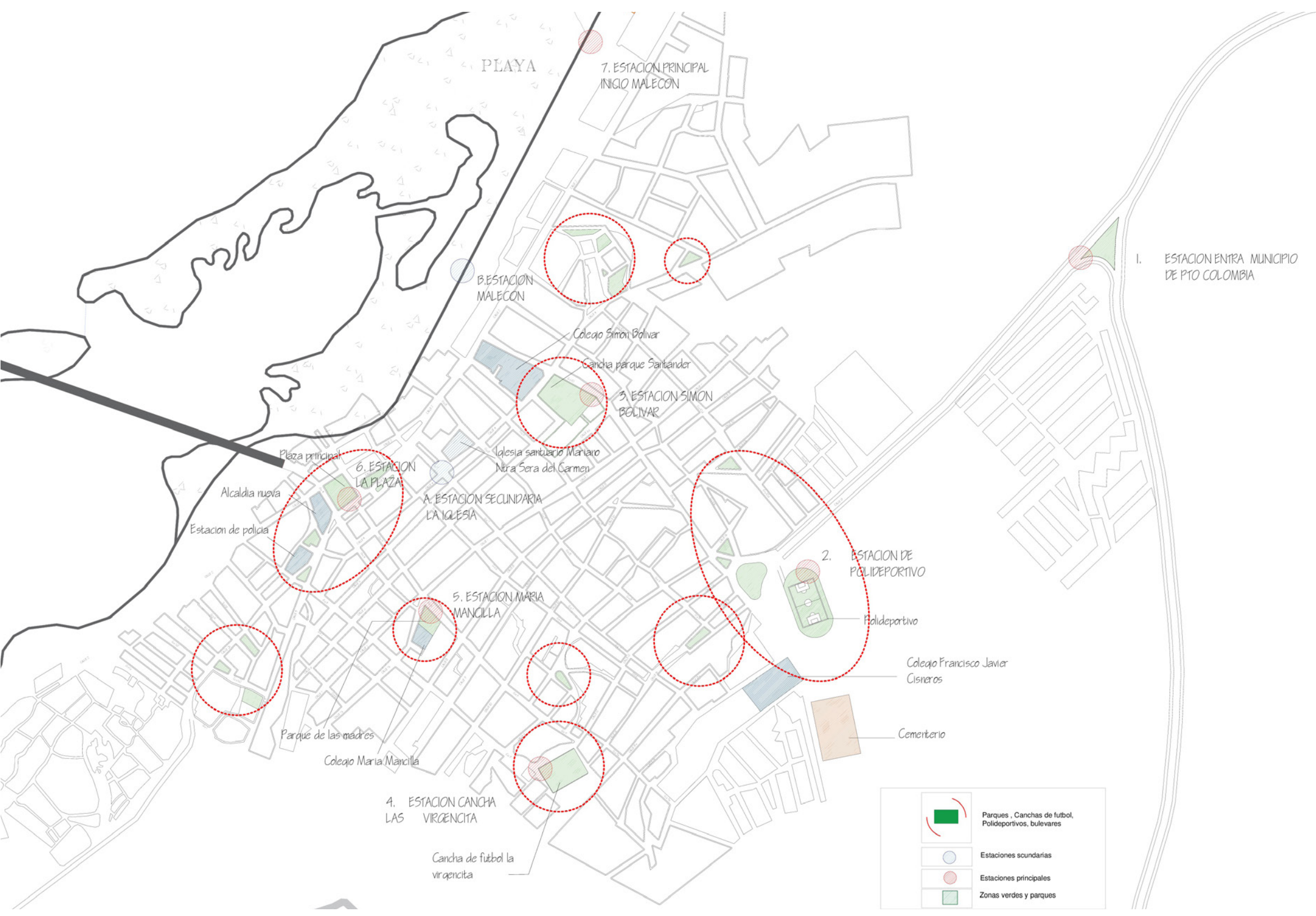

Fig. 5. Plano de ciclo-carriles y estaciones.

Fuente: elaboración propia con base en datos del PBOT, 2017. 


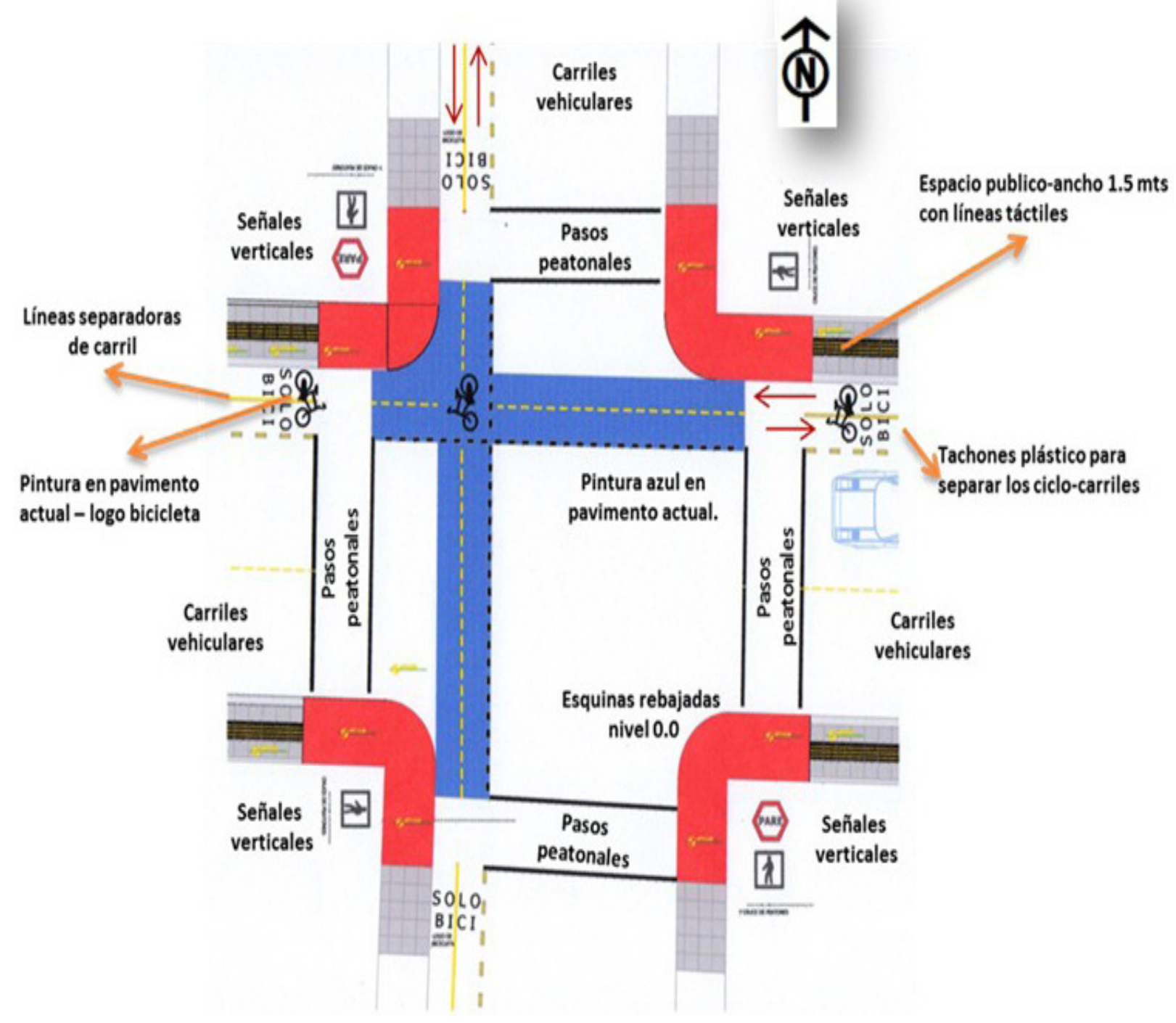

Fig. 6. Plano de intersecciones de ciclo-carriles.

Fuente: elaboración propia con base en datos del PBOT, 2017. 
En total se emplearán 255 bicicletas públicas y el mismo número de cicloparqueaderos, que deben cumplir lo que estipula el Manual de estacionamientos para bicicletas, guía de elección, servicio, integración y reducción de emisiones del Institute for Transportation \& Development Policy (ITDP, 2013). Las zonas de influencia de cada parque, plaza o terminal se determinaron con un radio de acción de 200 metros, arrojando un cubrimiento del $33 \%$ del área urbana del municipio, teniendo en cuenta que el municipio tiene 282 ha, correspondientes a $2.820 .235 \mathrm{~m}^{2}(100 \%)$ y las áreas de influencia de las estaciones suman 942.490 (33\%), restando un área por cubrir de $1.877 .745 \mathrm{~m}^{2}$ (67\%).

Es importante resaltar la participación que debería tener la bicicleta en un sistema intermodal de transporte en el área metropolitana de Barranquilla. La bicicleta se plantea como un medio sostenible de transporte para el municipio de Puerto Colombia, sin embargo, no se puede ocultar la necesidad de agrupar la bicicleta a un sistema intermodal de transporte en el municipio y en el área metropolitana de la capital del Atlántico. La clave de la intermodalidad es la integración entre cada uno de los modos de transporte. Así, los usuarios eligen, según sus necesidades, cómo hacer cada recorrido. La integración en una sola red permite viajar de manera segura, cómoda, y eficiente (Martínez, s.f.). De esta forma, la intermodalidad del transporte permite el beneficio y comodidad del usuario y, sobre todo, la conexión de cada medio de transporte. Sería importante y necesario la implementación, en un futuro, de sistemas intermodales para la bicicleta pública en el municipio de Puerto Colombia y en el área urbana de la ciudad de Barranquilla.

Se propone utilizar bicicletas ecológicas de bambú, dada la ubicación del municipio que esta bordeado en el oriente por el mar Caribe y la alta salinidad que hay en el ambiente.

\section{Conclusiones}

Los resultados muestran la necesidad de los habitantes de contar con un sistema de transporte amigable con el medio ambiente, la salud y que sea gratuito. Este disminuirá la congestión vehicular y los accidentes que se presentan con los automotores. El sistema de bicicletas podrá ser utilizado por todas las personas que se inscriban en el programa de bicipúblicas del municipio, esto con el fin de llevar un control de las personas a las que se le presta la bicicleta y, de igual forma, todos los turistas. La implementación de este sistema de movilidad ayudará a desestimular el uso de la motocicleta, teniendo en cuenta que es el automotor que ha incrementado el índice de accidentalidad y mortalidad de las personas a nivel local y que, por otra parte, les brinda la posibilidad a las personas de desplazarse dentro del municipio totalmente gratis. 

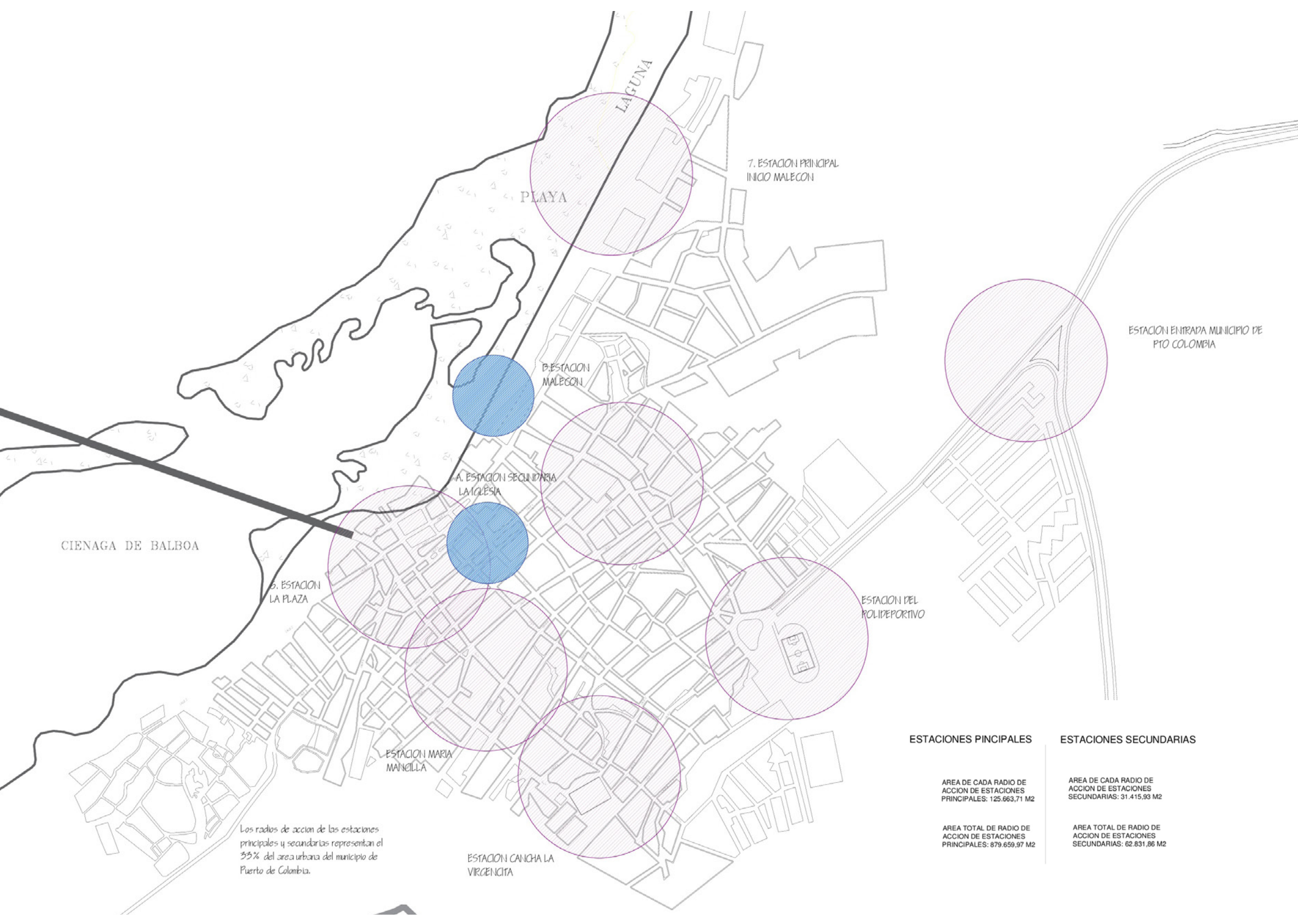

Fig. 7. Plano de radio de acción de las estaciones.

Fuente: elaboración propia con base en datos del PBOT, 2017. 


\section{REFERENCIAS}

Banco Mundial; DNP. (2012). Sistema de ciudades. Una aproximación visual al caso colombiano. Capítulo Transporte, congestión y movilidad. p. 45-52. Recuperado de https://colaboracion. dnp.gov.co/CDT/Vivienda\%20 Agua\%20y\%20Desarrollo\%20 Urbano/Sistema\%20de\%20 Ciudades-1\%20Introducción.pdf

Cantillo, V. (2011). Pautas para mejorar la circulación vehicular. Barranquilla: UniNorte.

Departamento Administrativo Nacional de Estadística-DANE. (2005). Censo nacional 2005. Recuperado de https://www.dane.gov.co/files/ censos/libroCenso2005nacional. pdf

European Commission-EC. (2013). Guidelines. Developing and implementing a sustainable urban mobility plan. Bruselas: Directorate-General for Mobility and Transport.

European Commission's. (2016). Transport sector economic analysis. Urban Mobility. Recuperado de https://ec.europa.eu/jrc/en/ research-topic/transport-sectoreconomic-analysis
García, O. (2018). Más allá de transporte: movilidad urbana sostenible. Recuperado de http://ieu.unal.edu.co/ noticias-del-ieu/item/del-transportea-la-movilidad-urbana-sostenible

ITDP. (2013).Manual de estacionamientos para bicicletas, guía de elección, servicio, integración y reducción de emisiones. New York: Institute for transportation \& development policy.

Jiménez, L. (s.f.). Nosotros: Lychnos. Recuperado de http://www.fgcsic. es/lychnos/es_ES/articulos/transporte_movilidad_claves_para_la_ sostenibilidad

Lizárraga, C. (2006). Movilidad urbana sostenible: un reto para las ciudades del siglo XXI. Economía, sociedad y territorio, $\mathrm{VI}(6), 283-321$.

López, L. (2018). La bicicleta como medio de transporte en la Movilidad Sustentable. México., D.F. Dirección General de Análisis Legislativo. Recuperado de http://bibliodigitalibd.senado.gob.mx/bitstream/ handle/123456789/3971/CA_23. pdf?sequence $=1$ \&isAllowed $=\mathrm{y}$

Martínez, M. (s.f.). ¿Qué es la intermodalidad urbana? La bicikleta. Recuperado de https://labicikleta.com/ la-intermodalidad-urbana/ 
Montezuma, R. (2003). Ciudad y transporte: La movilidad urbana. En M. Balbo, R. Jordán y D. Simioni, La ciudad inclusiva. Cuadernos de la Cepal (pp. 154-173). Santiago de Chile: Naciones Unidas.

Organización Mundial de la Salud-OMS. (2015). Informe sobre la situación mundial de la seguridad vial 2015. OMS. Recuperado de http://www. who.int/violence_injury_prevention/road_safety_status/2015/ Summary_GSRRS2015_SPA.pdf

República de Colombia. Agencia Nacional de Segguridad Vial. (2017). Observatorio Nacional de Seguridad Vial. Recuperado de http://ansv.gov.co/observatorio/ public/archivos/PLANESTRATEGICOINSTITUCIONAL10042018V1001docx.docx

República de Colombia. Concejo Municipal de Puerto Colombia. (2016). Plan de Desarrollo del Municipio de Puerto Colombia 2016-2019. Recuperado de http://puertocolombia-atlantico. gov.co/MiMunicipio/ProgramadeGobierno/Plan\%20de\%20 Desarrollo\%20Vigencia\%20 2016-2019.pdf
República de Colombia. Alcaldia municipal de Puerto Colombia. (2017). PBOT-Plan Básico de Ordenamiento Territorial de Puerto Colombia. Recuperado de https://mega.nz/\#F!ArAFx RRJ!TsYvdM2bozADQj1Y0OaMtQ

República de Colombia. Concejo colombiano de Seguridad; Mintransporte (2013). Plan Nacional de Seguridad Vial (PNSV) 2013-2021. Mintransporte. Recuperado de https://culturavial.files.wordpress.com/2014/01/ consulta_plan_nacional_de_ seguridad_vial_colombia_20132021.pdf

Reyes, E. (2018). La interfaz urbana. Una aproximación. On The W@terfront, 60(7). 3-40. Recuperado de http:// revistes.ub.edu/index.php/waterfront/ article/viewFile/21652/23397

Rojas, E. y Osorio, H. (2013). Andar: Movilidad Sostenible. Módulo Arquitectura-CUC, 12(1). 27-44. Recuperado de https://revistascientificas. cuc.edu.co/moduloarquitecturacuc/ article/view/37

Suero, D. (2010). Factibilidad del uso de la bicicleta como medio de transporte en la ciudad de Bogotá. AVANCES Investigación en Ingeniería, 12, 54-62. Recuperado de http://revistas. unilibre.edu.co/index.php/avances/ article/download/2675/2102/ 
Velásquez, C. (2015). Espacio público y movilidad urbana: Sistemas integrados de transporte masivo (SITM). [Tesis doctoral]. Programa de doctorado en Espacio Público y Regeneración Urbana, Facultad de Bellas Artes, Universitat de Barcelona, España. Recuperado de https://tdx.cat/handle/10803/319707
Universidad del Norte (2015). Lineamientos estratégicos y plan de acción para la competitividad del municipio de Puerto Colombia. Un norte para Puerto Colombia. Barranquilla: Uninorte. Recuperado de https://www.uninorte.edu. co/documents/5564742/eb8b8b9ac81d-42ef-891f-b0d7a34baf53 